\title{
L'importance de l'immigration pour le système de soins
}

\section{Stefanie Hostettler ${ }^{a}$, Esther Kraft ${ }^{b}$}

a Dr sc. EPFZ, division Données, démographie et qualité (DDQ) de la FMH

b lic. rer. oec., cheffe de la division Données, démographie et qualité (DDQ) de la FMH

\section{Statistique médicale de la FMH - un savoir fondé sur des données}

Combien y a-t-il de médecins en Suisse? Et qu'en est-il des femmes et des médecins étrangers? Depuis 1934, la statistique médicale annuelle de la FMH répond à ces questions et à bien d'autres en se fondant sur une base de données détaillée (cf. paragraphe «Base de données») et permet ainsi de suivre l'évolution du système de santé en Suisse. Grâce à ces nombreuses informations, il est possible d'identifier des corrélations, de reconnaître les éventuelles lacunes et de fixer des priorités pour répondre aux besoins actuels et à venir de la population suisse en matière de santé.

\section{Evolution de l'effectif médical}

En 2015, 35325 médecins (14268 femmes, 21057 hommes) exerçaient en Suisse, soit 977 de plus que l'année précédente. Cette augmentation est principalement due à la progression du nombre de femmes médecins (cf. fig. 1). Bien que les hommes restent plus nombreux que les femmes avec 59,6\%, la part des femmes a davantage progressé que celle des hommes au cours des dernières années. Entre 2010 et 2015, le nombre de femmes a augmenté de 31,6\%, et de seulement 8,4\% pour les hommes. En médecine humaine, le nombre de diplômés de sexe

$\mathrm{ch} /$ themen/

berufe/13930/13936/index html?lang=fr

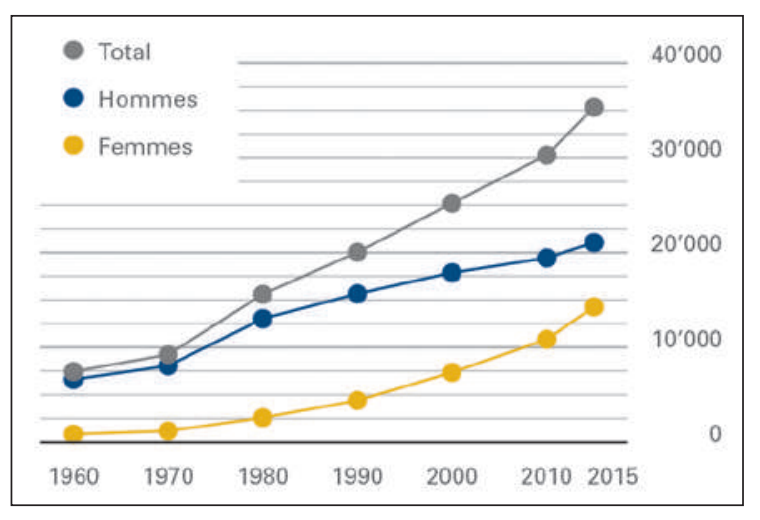

Figure 1: Nombre de médecins d'après l'année et le sexe 1960-2015.

féminin est majoritaire depuis déjà dix ans et atteint 55,7\% (femmes 515, hommes 409) [1]. La proportion de femmes est de $59,6 \%$ pour les octrois de titres fédéraux de spécialiste reconnus (1743 au total).

$51,3 \%$ des médecins exercent dans le secteur ambulatoire, $47,1 \%$ dans le secteur hospitalier et 1,6\% dans un autre secteur (cf. tab. 1). Avec 45,4\%, la part des femmes est la plus forte dans le secteur hospitalier, suivie de $36,1 \%$ dans le secteur ambulatoire et de $29,8 \%$ dans le troisième secteur.

Par rapport à 2010, le secteur hospitalier a progressé davantage (+21,3\%) que le secteur ambulatoire $(+12,7 \%)$. Si les médecins travaillent en majorité dans un seul secteur, ils sont $11 \%$ à exercer dans plus d'un secteur. La situation est à peu près la même pour le lieu de travail: environ $86,6 \%$ exercent dans un seul lieu, $11 \%$ dans deux lieux et 2,4\% dans trois lieux de travail ou plus.

Données et démographie - La statis-

\section{tique médicale 2015 de la FMH}

La division Données, démographie et qualité (DDQ) publie tous les ans les chiffres actuels et les tendances concernant la démographie du corps médical. Vous trouverez les statistiques 1940 à 2015, le moteur de recherche et de plus amples informations à ce sujet sur le site Internet de la FMH (www. fmh.ch $\rightarrow$ Services $\rightarrow$ Statistique). La division DDQ se tient volontiers à votre disposition pour tout complément d'information: ddq[at]fmh.ch / 0313591111. 
Tableau 1: Aperçu du nombre de médecins en exercice par secteur (activité principale) en 2015

\begin{tabular}{|c|c|c|c|c|c|c|}
\hline \multirow[b]{2}{*}{ Secteur ambulatoire } & \multicolumn{2}{|c|}{ Femmes } & \multicolumn{2}{|c|}{ Hommes } & \multicolumn{2}{|l|}{ Total } \\
\hline & 6546 & $36,1 \%$ & 11582 & $63,9 \%$ & 18128 & $51,3 \%$ \\
\hline Secteur hospitalier & 7554 & $45,4 \%$ & 9080 & $54,6 \%$ & 16634 & $47,1 \%$ \\
\hline Total & 14268 & $40,4 \%$ & 21057 & $59,6 \%$ & 35325 & $100 \%$ \\
\hline
\end{tabular}

\section{Densité médicale}

En moyenne, on compte 4,2 médecins pour 1000 habitants en Suisse. Les cantons de Bâle-Ville, de Genève et de Zurich comptent la densité médicale la plus élevée, avec respectivement 10, 6,2 et 5 médecins pour 1000 habitants, et les cantons d'Uri, d'Appenzell Rhodes-Intérieures et d'Obwald la plus basse, avec 1,6, 1,8 et 2,1 médecins pour 1000 habitants. La densité médicale des cantons suisses est décrite à la figure 2 .

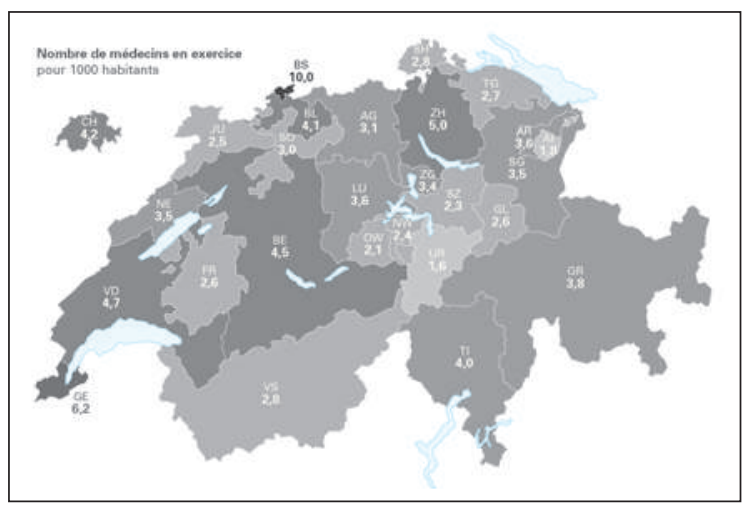

Figure 2: Nombre de médecins par 1000 habitants et par canton (source FMH - Statistique médicale 2014 et OFS - Statistique de la population).

La figure 3 montre la densité médicale pour 10000 habitants dans le secteur ambulatoire en fonction de la typologie des communes pour les médecins de premier recours et les spécialistes. Dans les communes urbaines, la densité médicale est nettement plus élevée que dans les communes rurales. Dans les centres urbains, la densité de spécialistes est près du double de celle des médecins de premier recours, alors que la densité des médecins de premier recours dépasse celle des spécialistes dans les communes rurales.

\section{Pyramide des âges}

En augmentation depuis plusieurs années, la moyenne d'âge des médecins en Suisse se situe aujourd'hui à 49 ans (cf. tab. 2), alors qu'elle était de 45,8 ans il y a dix ans. Un médecin du secteur ambulatoire a en moyenne 54,4 ans, soit dix ans de plus que son collègue du secteur hospitalier, qui a 43 ans. Cette différence

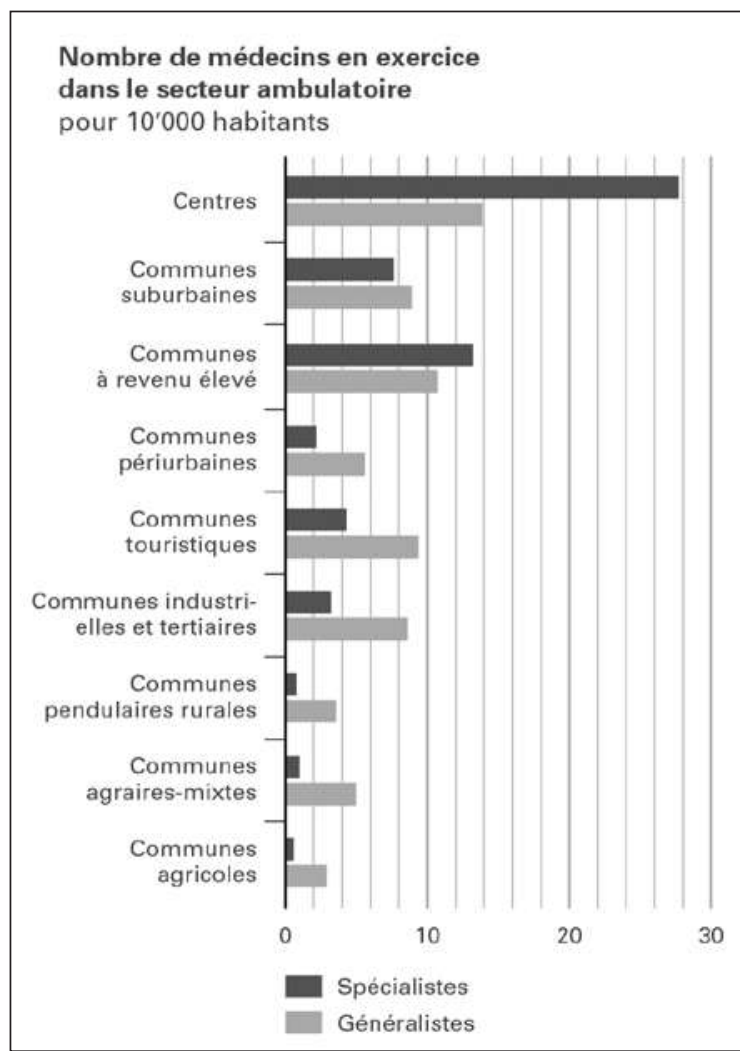

Figure 3: Médecins de premier recours et spécialistes en exercice pour 10000 habitants et par type de commune en 2014 (source FMH - Statistique médicale 2014 et OFS Statistique de la population).

d'âge entre le secteur ambulatoire et le domaine hospitalier s'explique principalement par le fait que la formation postgraduée des assistants se déroule essentiellement en milieu hospitalier. En 2015, les médecins avaient 36,6 ans en moyenne ( 36 pour les femmes, 37,6 pour les hommes) au moment de l'obtention de leur titre de spécialiste, autrement dit au terme de leur formation postgraduée.

La figure 4 montre qu'en dessous de 40 ans, le nombre de femmes médecins est majoritaire dans les deux secteurs. A partir de 40 ans, le nombre d'hommes en exercice dépasse actuellement celui des femmes. Mais la hausse de la proportion de femmes chez les étudiants devrait se refléter dans les différentes tranches d'âge au cours des prochaines années. 
Tableau 2: Age moyen du corps médical en 2015.

\begin{tabular}{llll} 
& Femmes & Hommes & Total \\
\hline Secteur ambulatoire & 51,2 & 56,1 & 54,4 \\
\hline Secteur hospitalier & 39,9 & 45,6 & 43,0 \\
\hline Autre secteur & 45,2 & 51,6 & 49,0
\end{tabular}

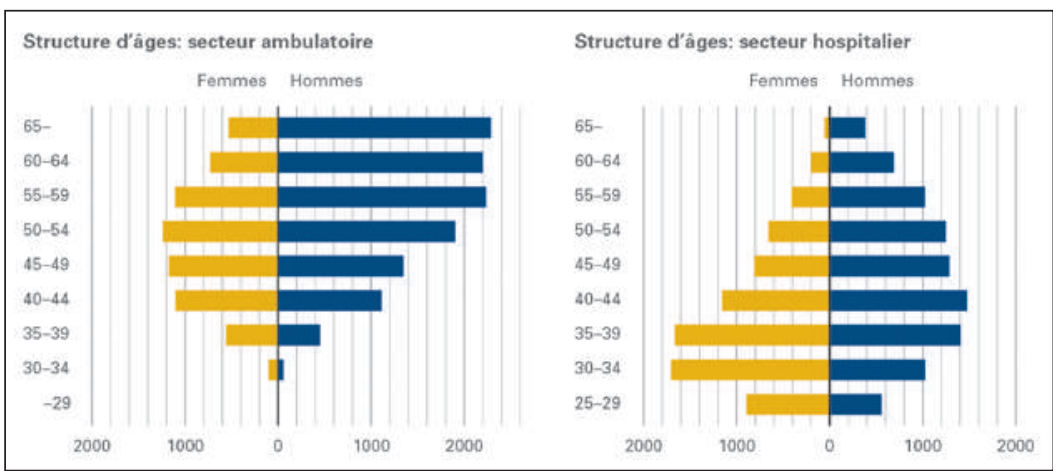

Figure 4: Pyramide des âges et répartition sexo-spécifique des médecins en exercice par secteur en 2015. médecins étrangers proviennent d'Allemagne $(17,7 \%)$, d'Italie (2,6\%), d'Autriche (1,9\%) et de France (1,8\%).

Sur les 1743 titres de spécialiste décernés en 2015, 41\% l'ont été à des médecins titulaires d'un diplôme étranger.

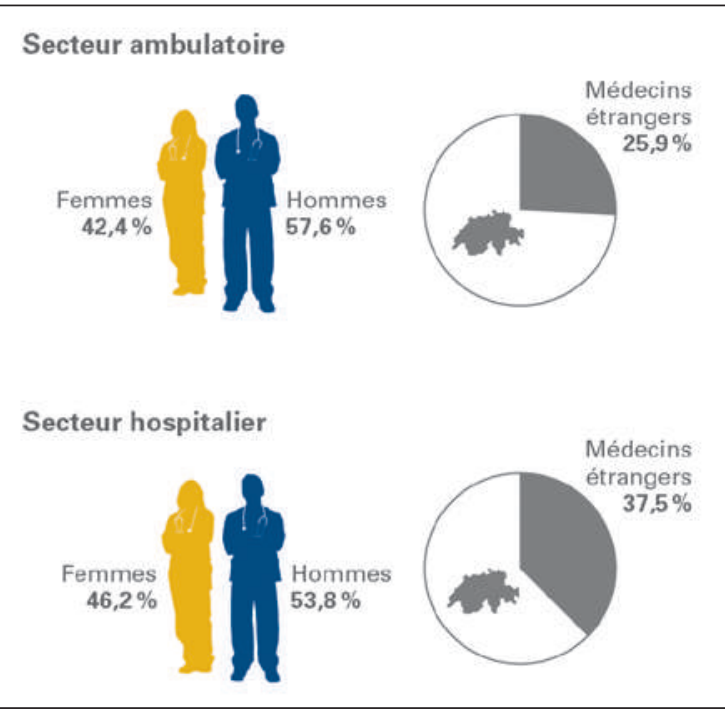

Figure 5: Part de médecins étrangers par secteur et sexe en 2015.

La spécialisation la plus représentée est la médeci interne générale (23,6\%) (cf. tab. 3), suivie de la psychiatrie et psychothérapie $(10,2 \%)$, de la gynécologie et obstétrique (4,9\%), de la pédiatrie $(4,8 \%)$ et de l'anesthésiologie (4,2\%). 4877 médecins - dont 1296 femmes ont obtenu plusieurs titres de spécialiste. 3741 porteurs de plusieurs titres ont obtenu le titre de spécialiste en médecine interne générale.

On trouve le plus grand nombre de femmes médecins en psychiatrie et psychothérapie d'enfants et d'adolescents $(63,1 \%)$, en pédiatrie $(59,8 \%)$ et en gynécologie et obstétrique (57,9\%). Le nombre d'hommes est supérieur à celui des femmes dans les spécialisations chirurgicales (chirurgie orale et maxillo-faciale 93,1\%, chirurgie thoracique $93,1 \%$, chirurgie orthopédique $91,7 \%$, chirurgie vasculaire $89,9 \%$ ).

\section{Diplômes de médecin étrangers}

11138 médecins exerçant en Suisse, soit 31,5\%, viennent de l'étranger (c'est-à-dire qu'ils ont obtenu leur diplôme de médecine à l'étranger). Dans le secteur ambulatoire, la part de médecins étrangers se monte à $25,9 \%$ alors qu'elle est de 37,5\% dans le secteur hospitalier. Par rapport à l'année précédente, la part de médecins étrangers a augmenté de $1 \%$ et a passé de $30,5 \%$ à $31,5 \%$. Avec respectivement $42,4 \%$ et $46,2 \%$, la part de femmes au bénéfice d'un diplôme de médecin étranger est légèrement plus basse que celle des hommes dans les deux secteurs (57,6\% et 53,8\%) (cf. fig. 5). La majorité des

\section{Fonction}

Parmi les 18128 médecins en exercice dans le secteur ambulatoire, 83,6\% sont propriétaires ou propriétaires associés de leur cabinet médical. 4,1\% des médecins sont engagés en tant qu'assistant ou spécialiste au cabinet médical. Le reste des médecins de ce secteur exerce une autre fonction. Les médecins en formation postgraduée (52,2\% environ) représentent le groupe le plus important du secteur hospitalier. Comme on peut s'y attendre, le nombre de médecins tend à diminuer plus le degré hiérarchique est élevé. On compte par ex. $19,3 \%$ de chefs de clinique, $12 \%$ de médecins adjoints et 9,3\% de médecins-chefs. Le nombre de femmes prédomine uniquement chez les médecins-assistants $(58,1 \%)$ et ne fait que diminuer ensuite pour atteindre $44,8 \%$ chez les chefs de clinique, $22,2 \%$ chez les médecins adjoints et $11,9 \%$ chez les médecins-chefs.

\section{Taux d'occupation}

Les indications concernant le taux d'occupation, et toutes les évaluations figurant dans ce second volet, se fondent sur les données auto-déclarées par les médecins au moyen d'un questionnaire sur le portail des membres myFMH (échantillon myFMH, cf. paragraphe «Base de données»). 
Tableau 3: Aperçu du nombre de médecins par spécialisation (activité principale) en 2015.

\begin{tabular}{|c|c|c|c|}
\hline & Femmes & Hommes & Total \\
\hline Allergologie / immunologie & 47 & 99 & 146 \\
\hline Anesthésiologie & 619 & 849 & 1468 \\
\hline Angiologie & 42 & 139 & 181 \\
\hline Cardiologie & 116 & 586 & 702 \\
\hline Chirurgie card. et vasc. thorac. & 8 & 71 & 79 \\
\hline Chirurgie orale + maxillo-faciale & 6 & 81 & 87 \\
\hline Chirurgie & 223 & 884 & 1107 \\
\hline Chirurgie de la main & 40 & 144 & 184 \\
\hline Chirurgie orthopédique & 88 & 969 & 1057 \\
\hline Chirurgie pédiatrique & 30 & 49 & 79 \\
\hline Chirurgie plastique & 51 & 137 & 188 \\
\hline Chirurgie thoracique & 2 & 27 & 29 \\
\hline Chirurgie vasculaire & 7 & 62 & 69 \\
\hline Dermatologie et vénéréologie & 250 & 283 & 533 \\
\hline Endocrinologie / diabétologie & 84 & 116 & 200 \\
\hline Gastroentérologie & 45 & 300 & 345 \\
\hline Génétique médicale & 15 & 10 & 25 \\
\hline Gynécologie et obstétrique & 997 & 725 & 1722 \\
\hline Hématologie & 59 & 115 & 174 \\
\hline Infectiologie & 59 & 110 & 169 \\
\hline Médecine physique et réadaptation & 64 & 144 & 208 \\
\hline Médecin praticien & 643 & 708 & 1351 \\
\hline Médecine du travail & 44 & 76 & 120 \\
\hline Médecine intensive & 170 & 370 & 540 \\
\hline Médecine interne générale & 3114 & 5214 & 8328 \\
\hline Médecine légale & 22 & 28 & 50 \\
\hline Médecine nucléaire & 14 & 55 & 69 \\
\hline Médecine pharmaceutique & 22 & 34 & 56 \\
\hline Médecine tropicale et méd. voy. & 6 & 21 & 27 \\
\hline Néphrologie & 69 & 125 & 194 \\
\hline Neurochirurgie & 21 & 149 & 170 \\
\hline Neurologie & 183 & 387 & 570 \\
\hline Neuropathologie & 2 & 11 & 13 \\
\hline Oncologie médicale & 124 & 204 & 328 \\
\hline Ophtalmologie & 390 & 591 & 981 \\
\hline $\mathrm{ORL}$ & 126 & 362 & 488 \\
\hline Pathologie & 120 & 125 & 245 \\
\hline Pédiatrie & 1020 & 687 & 1707 \\
\hline Pharmacologie et toxicologie cliniques & 18 & 22 & 40 \\
\hline Pneumologie & 56 & 227 & 283 \\
\hline Prévention et santé publique & 39 & 37 & 76 \\
\hline Psychiatrie d'enfant & 406 & 237 & 643 \\
\hline Psychiatrie et psychothérapie & 1509 & 2074 & 3583 \\
\hline Radiologie & 234 & 591 & 825 \\
\hline Radio-oncologie / radiothérapie & 41 & 72 & 113 \\
\hline Rhumatologie & 122 & 341 & 463 \\
\hline Urologie & 39 & 282 & 321 \\
\hline Pas de réponse & 2862 & 2127 & 4989 \\
\hline Total & 14268 & 21057 & 35325 \\
\hline
\end{tabular}

En 2015, les médecins ont travaillé en moyenne 8,9 demi-journées par semaine (cf. tab. 4). Dans le secteur ambulatoire, le taux d'activité moyen était de 8,3 de- mi-journées, soit plus d'une demi-journée de moins que dans le secteur hospitalier (9,6 demi-journées). Le taux d'occupation moyen des femmes médecins (en ambulatoire: 6,9; en hospitalier: 8,9 demi-journées) est nettement inférieur à celui de leurs collègues masculins (en ambulatoire: 8,9; en hospitalier: 10,2 demi-journées).

Tableau 4: Taux d'occupation moyen par secteur et par sexe (en demi-journées hebdomadaires) en 2015 ( $n=8380$ ).

\begin{tabular}{llll} 
& Femmes & Hommes & Total \\
\hline Secteur ambulatoire & 6,9 & 8,9 & 8,3 \\
\hline Secteur hospitalier & 8,9 & 10,2 & 9,6 \\
\hline Autre secteur & 7,5 & 9,1 & 8,5 \\
\hline Total & 7,6 & 9,4 & 8,9 \\
\hline
\end{tabular}

En extrapolant les taux d'occupation moyens (2008 = 9,0; $2014=8,9$ demi-journées) à l'ensemble du corps médical et en les transposant en équivalents plein temps (EPT), on obtient 26724 EPT en 2008 et 29843 en 2014. En tenant compte de l'évolution de la population (2008 = 7701856; 2014 = 8236573), la densité médicale moyenne était de 3,5 médecins pour 1000 habitants en 2008, et de 3,6 en 2014. En d'autres termes, la densité médicale est restée à peu près la même au cours des sept dernières années. En comparant l'évolution dans le secteur ambulatoire et hospitalier, on constate que la densité de spécialistes dans le secteur hospitalier a progressé davantage (fig. 6).

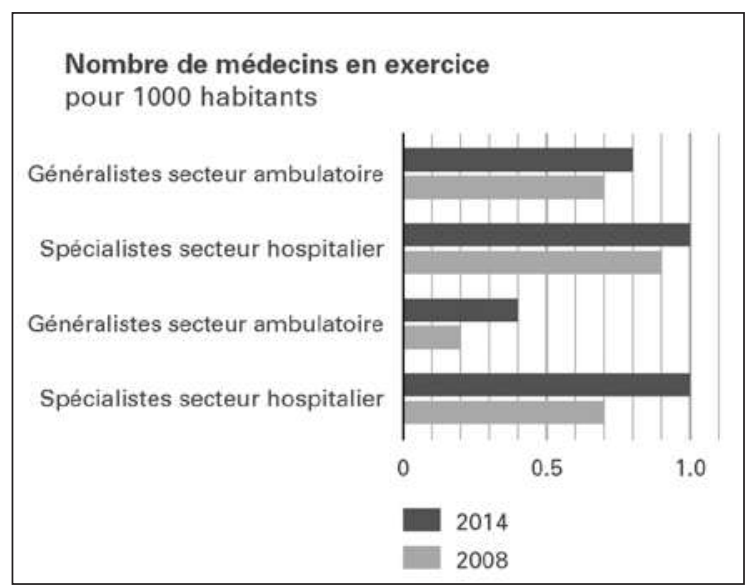

Figure 6: Densité médicale en équivalents plein temps pour 1000 habitants et par secteur de soins - comparaison 2008 et 2014 (source FMH - Statistique médicale 2008 et 2014, et OFS - Statistique de la population).

\section{Structures de cabinets et réseaux de médecins}

Dans le secteur ambulatoire, les médecins exercent principalement en cabinet individuel (56,1\%). Depuis 2008 , ce taux a diminué de $6 \%$ environ. La moitié des femmes environ $(47,3 \%)$ exercent dans un cabinet indi- 
viduel, et 52,7\% dans un cabinet double ou un cabinet de groupe. Chez les hommes, la part de médecins dans un cabinet individuel est légèrement plus élevée à $60,7 \%$. Enfin, un cabinet de groupe compte en moyenne 4,1 médecins.

Selon les données auto-déclarées, 49,2\% des médecins exerçant dans le domaine ambulatoire sont affiliés à un réseau de médecins. Alors que 69,2\% d'hommes médecins sont dans un réseau, les femmes sont 30,6\% à y être affiliées.

\section{Identifier les enjeux - développer des solutions pérennes}

Alors que la part de médecins étrangers a continué sa progression en 2015, la question de la dépendance de notre système de santé envers les professionnels étrangers se pose. En outre, on note un certain déséquilibre entre la répartition des spécialisations (surabondance de médecins spécialistes, pénurie de médecins de premier recours) et dans les régions (surabondance de l'offre dans les centres urbains, pénurie dans les régions rurales) [2]. D’après le baromètre des consommateurs suisses alémaniques "Pulsmesser», les coûts de la santé figurent en première place des préoccupations des consommateurs, puisque neuf personnes interrogées sur dix déclarent être préoccupées par les coûts de santé [3]. L'espérance de vie élevée (avec 82,9 ans, la Suisse affiche l'espérance de vie la plus élevée de toute l'Europe [4]), l'augmentation des maladies chroniques et les progrès médicotechniques notamment ont un impact important sur les conditions permettant de continuer à garantir et assurer la prise en charge de la population sur l'ensemble du territoire.

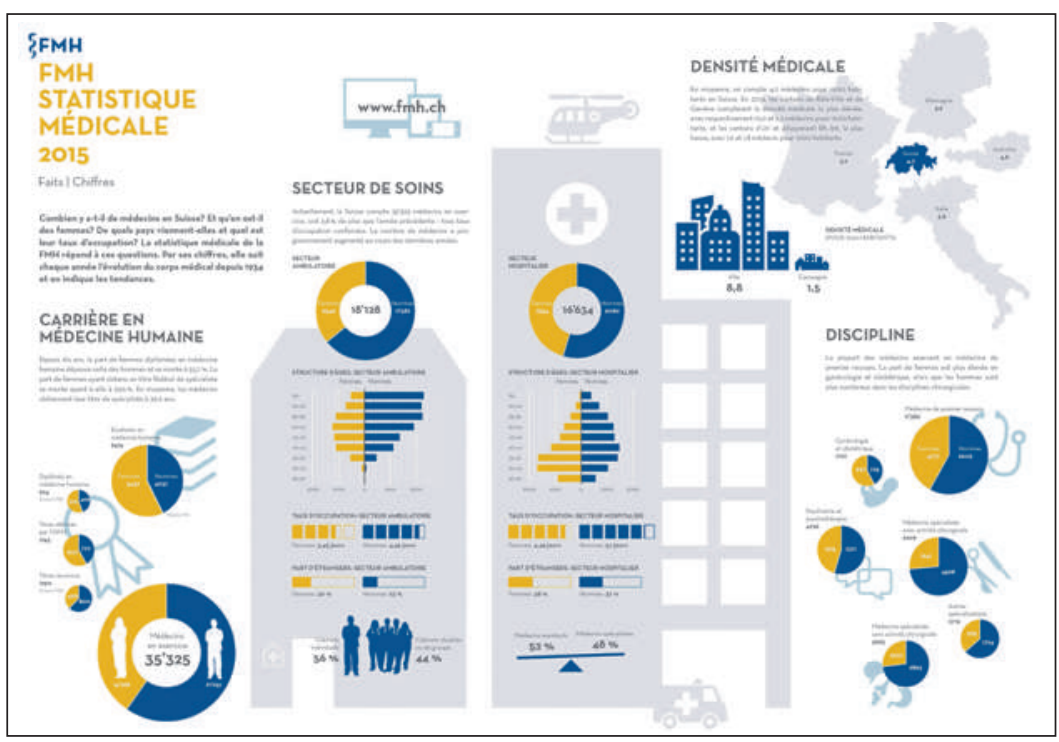

Figure 7: Poster de la statistique médicale 2015 de la FMH.
Pour cela, il est primordial de mettre l'accent sur la qualité des soins médicaux et des résultats pour les patients, dans le but de fournir une prise en charge adaptée et de pallier le sous- ou le sur-approvisionnement. Seules des connaissances approfondies de la situation et le développement de solutions pérennes permettront d'atteindre cet objectif.

La FMH s'engage depuis de nombreuses années pour des données de qualité élevée. La statistique médicale de la FMH encourage le transfert de connaissances et fournit une importante contribution à l'analyse de la situation des soins en Suisse. A cet effet, la FMH a également lancé d'autres projets visant par exemple à déterminer le nombre de médecins qui ont abandonné leur activité médicale (et les raisons de cette réorientation professionnelle), à élaborer des itinéraires de soins intersectoriels ou encore à examiner la qualité de l'indication. Le monde politique et les différents acteurs du domaine de la santé ont pour mission de prendre les décisions qui s'imposent en se fondant sur des données scientifiques tout en faisant preuve d'efficacité et de cohérence, et à fixer les priorités devant permettre de garantir à long terme la sécurité et la qualité du système de santé suisse.

\section{Base de données}

La statistique médicale de la FMH est établie en fin d'année (jour de référence: 31 décembre de l'année concernée). La banque de données de la FMH ( $\mathrm{n}=35278$ ) contient les principales caractéristiques de la démographie médicale telles que l'âge, le sexe, la nationalité et le lieu où le médecin exerce sa profession. Les données concernant l'activité professionnelle (taux d'activité, structures des cabinets, etc.) reposent sur les données auto-déclarées recueillies au moyen d'un questionnaire sur le portail des membres myFMH (échantillon myFMH). L'échantillon myFMH contient les données de plus de 13600 médecins qui ont été comparées avec les données de l'ensemble de la population de médecins en exercice en Suisse (sexe et secteur). On a ainsi constaté que le secteur hospitalier et les femmes du secteur hospitalier étaient sous-représentés dans l'échantillon (différence $>10 \%$ par rapport à l'ensemble de la population). Pour minimiser ce biais, les chiffres par secteur et par sexe ont été analysés séparément ou pondérés en conséquence.

\section{Poster - clair et compact}

Si vous souhaitez avoir un aperçu de la statistique médicale de la FMH sous forme compacte, n'hésitez pas à commander notre poster à l'adresse www.fmh.ch $\rightarrow$ Services $\rightarrow$ Statistique ou par courriel à ddq[at]fmh.ch. 


\section{Glossaire (par ordre alphabétique)}

Activité principale

Autre secteur

Est considéré comme activité principale le secteur dans lequel le médecin exerce la majeure partie de son activité.

\begin{tabular}{|c|c|}
\hline & $\begin{array}{l}\text { ni du secteur hospitalier, par ex. professeurs, privat-docents, médecins engagés par des } \\
\text { assurances, etc. }\end{array}$ \\
\hline $\begin{array}{l}\text { Cabinet à deux médecins } \\
\text { ou de groupe }\end{array}$ & Utilisation d'appareils, d'équipements ou de locaux par deux ou plusieurs médecins. \\
\hline Cabinet individuel & Utilisation d'appareils, d'équipements ou de locaux par un seul médecin. \\
\hline Canton & $\begin{array}{l}\text { Le canton pris en compte est celui dans lequel le médecin exerce son activité principale. S'il } \\
\text { n'a rien indiqué à ce sujet, c'est le canton de l'adresse de contact qui est pris en compte. }\end{array}$ \\
\hline Discipline principale & $\begin{array}{l}\text { La discipline principale d'un médecin correspond au titre de spécialiste dans le cadre duquel } \\
\text { il exerce la majeure partie de son activité médicale (d'après son auto-déclaration et les règles } \\
\text { prédéfinies). }\end{array}$ \\
\hline Médecins du secteur ambulatoire & Médecins qui exercent leur activité principale dans le secteur ambulatoire. \\
\hline Médecins du secteur hospitalier & Médecins qui exercent leur activité principale en milieu hospitalier. \\
\hline Médecins d'un autre secteur & $\begin{array}{l}\text { Médecins qui n'exercent leur activité principale ni dans le secteur ambulatoire ni en milieu } \\
\text { hospitalier. }\end{array}$ \\
\hline Médecins en exercice & $\begin{array}{l}\text { Les médecins en exercice comprennent les personnes ayant achevé avec succès des études } \\
\text { de médecine de niveau universitaire, disposant ou non d'une formation postgraduée et exer- } \\
\text { çant dans le secteur ambulatoire, dans le secteur hospitalier (médecins-assistants compris) } \\
\text { ou dans un autre secteur (par ex. administration, assurances, etc.) (source: OFS). }\end{array}$ \\
\hline Réseaux de médecins & $\begin{array}{l}\text { Les réseaux de médecins sont des organisations formées par des prestataires de soins et } \\
\text { assurant la couverture médicale. Sur la base d'une collaboration contractuelle tant mutuelle } \\
\text { qu'avec des fournisseurs et des organismes extérieurs au réseau, ils fournissent des presta- } \\
\text { tions de santé axées sur les besoins des patients. Cette collaboration repose sur un proces- } \\
\text { sus thérapeutique convenu par contrat, des structures organisationnelles entrepreneuriales } \\
\text { et une culture commune de la prise en charge des patients. }\end{array}$ \\
\hline
\end{tabular}

Secteur ambulatoire

Font partie du secteur ambulatoire les consultations données et les soins dispensés par des médecins en cabinet individuel ou de groupe. Les patients sont généralement traités de manière ambulatoire ou à leur domicile (visites à domicile). Font également partie du secteur ambulatoire les activités des médecins consultants privés dans les hôpitaux ou dans des dispensaires, des infirmeries ou des établissements analogues attachés à des entreprises, des écoles, des homes pour personnes âgées, des organisations syndicales et des confréries (cabinet médical indépendant ou semblable) (source: OFS).

Secteur hospitalier

Font partie du secteur hospitalier les traitements médicaux, diagnostics, soins, interventions chirurgicales, analyses, services d'urgence et activités de formation prégraduée, postgraduée et continue, etc. dans les hôpitaux. Font également partie du secteur hospitalier les foyers protégés avec un encadrement social $24 \mathrm{~h}$ sur 24 accueillant des enfants, des personnes âgées et des groupes de personnes dont l'autonomie est limitée (source: OFS).

Taux d'occupation

Le taux d'occupation est indiqué en demi-jours. Un demi-jour correspond à un volume de travail de 4 à 6 heures. Un emploi à plein temps correspond à un taux d'occupation hebdomadaire moyen de 10 demi-jours.

Correspondance: FMH/division DDQ Elfenstrasse 18 CH-3000 Berne 15 Tél. 0313591111 ddq[at]fmh.ch
Tous les traitements qui ne sont pas réputés hospitaliers ou semi-hospitaliers.

Traitements ambulatoires en milieu hospitalier

Traitements hospitaliers en milieu hospitalier
Séjours à l'hôpital d'au moins 24 heures pour des examens, des traitements et des soins. Séjours à l'hôpital de moins de 24 heures, au cours desquels un lit est occupé durant une nuit. Séjours à l'hôpital en cas de transfert dans un autre hôpital ou en cas de décès. 